Pediat. Res. 4: 229-237 (1970)

Bleeding time coagulation

newborns fibrin

\title{
Altered Platelet Function in Newborns
}

\author{
Marilyn M. Mull ${ }^{[36]}$ and William E. Hathaway \\ Department of Pediatrics, University of Colorado Medical Center, Denver, Colorado, USA
}

\begin{abstract}
Extract
Studies of platelet function were performed on venous blood specimens from 64 newborn infants within $0-48 \mathrm{~h}$ after birth. Fifty-two of the infants studied were normal, full-term average for gestational age (AGA) infants, three were normal, premature AGA infants, seven were premature AGA infants with the respiratory distress syndrome (RDS), and two were premature AGA infants who had repeated apneic periods. Although it was not possible to study all variables of platelet function in each of the groups, those studies that were performed showed no differences in the results based on the group to which an individual infant belonged.

Compared with 24 normal adult controls, the infants showed impaired platelet aggregation in the presence of ADP, collagen, and thrombin. The mean time required for maximal platelet aggregation with ADP, collagen, and thrombin for the adults was 120,230 , and $74 \mathrm{sec}$, respectively, compared with values of 160,443 , and $158 \mathrm{sec}$ for the infants (table II, figs. 2, 3, and 4). The percentage total platelet aggregation with ADP, collagen, and thrombin for the adults was 79, 91 , and $74 \%$ compared with 47,73 , and $47 \%$, respectively, for the infants (table II, figs. 2, 3, and 4). Infant whole blood and platelet-rich plasma clot retraction were markedly defective compared with normal adults (table I). In addition, platelet factor 3 release in 10 normal infants, as measured by the modified Stypven time, was decreased when compared with 10 values for normal adults (fig. 5). Other variables of platelet function (bleeding times, native blood platelet adhesiveness, petechiometer tests) studied in newborn infants were normal (table I). Thrombelastograms were compatible with hypercoagulability of the blood (table I). None of the infants studied had clinical evidence of a bleeding tendency.

In vitro mixing experiments suggested that the impaired ADP platelet aggregation was due neither to the presence of plasma ADP inhibitors, a refractory state due to increased circulating levels of ADP, anticoagulants, nor to a selected population of platelets. Physiologic alterations of infant platelets are similar to platelet functional changes observed in thrombasthenia.
\end{abstract}

\section{Speculation}

Transient physiologic alterations have been described in many components of the newborn's blood, i. e., decreased plasma coagulation factors, red cell enzyme changes, low immunoglobulins, and an altered kinin system. It is not surprising, therefore, to find a transient platelet functional deficiency as well. Indeed, this hypofunction may be a defense against intravascular coagulation at a time when the infant's blood may be hypercoagulable and susceptible to increased thrombotic tendencies. Whether this platelet functional alteration could aggravate or be related to the cause of hemorrhage in severely ill infants remains to be shown. 
Introduction

The increased incidence in infants of hemorrhage in the newborn period has been documented in several studies $[1,18,21]$. This predisposition to hemorrhage in newborns, particularly the ill or premature infant, has been attributed in part to perinatal hypoxia with resultant ischemic damage to blood vessels. In addition, newborns have been said to have an increased 'capillary fragility' [1]. Since modern concepts of hemostasis suggest that platelet function is a major determinant of small vessel integrity and hence 'capillary fragility', it was decided to examine whether variables of platelet function were altered in newborn infants compared with normal adults.

\section{Materials and Methods}

\section{Subjects}

Platelet function studies were performed on 64 infants within $0-48 \mathrm{~h}$ of life. Fifty-two of the newborns were normal, full-term average for gestational age (AGA) infants, three were normal, premature AGA infants, seven were premature, AGA infants with the respiratory distress syndrome (RDS), and two were premature, AGA infants who had repeated apneic periods. None of the infants was clinically jaundiced at the time studied. Identical platelet function studies were performed on 24 healthy adults between the ages of 25 and 35 years [23].

\section{Blood Collection}

Venous blood was obtained by a two-syringe technique using disposable 20 -gauge needles and plastic syringes. Native whole blood was drawn into the first syringe. The second syringe contained $0.1 \mathrm{ml}$ of anticoagulant ( 3 parts $0.1 \mathrm{~m}$ sodium citrate and 2 parts $0.1 \mathrm{~m}$ citric acid) per milliliter of whole blood withdrawn. The volume of anticoagulant was adjusted on the basis of the hematocrit determination [9]. Specimens of cord blood from 26 normal term infants were obtained by double clamping the umbilical cord shortly after delivery of the infant. Blood was then drawn immediately from the isolated umbilical vein. Plateletrich plasma ( $\mathrm{PRP}$ ) was prepared by centrifugation at $130 \times g$ for $20 \mathrm{~min}$ at $4^{\circ}$. The PRP was placed in siliconized glass or plastic tubes at room temperature. Platelet-free plasma (PFP) was obtained by recentrifuging the remainder of the original sample of citrated venous blood at $2,750 \times \mathrm{g}$ for $20 \mathrm{~min}$. The majority of tests were completed within $4 \mathrm{~h}$ after collection. All platelet counts were performed according to the phase method [2].

\section{Bleeding Time and Clot Retraction}

Bleeding time measurements were performed by a modified Ivy technique (normal 2-7 min) using the volar aspect of the forearm and a Dade hemolet. Whole blood clot retraction was determined qualitatively after placing $1.0 \mathrm{ml}$ of native whole blood in a clean new glass test tube and incubating in a water bath at $37^{\circ}$ for $24 \mathrm{~h}$. The PRP clot retractions were done by adding 4.0 units of bovine thrombin to $1.0 \mathrm{ml}$ PRP $\left(300,000\right.$ platelets $\left./ \mathrm{mm}^{3}\right)$. Clot retractions were graded $0-4+$.

\section{Petechiometer Test}

Petechiometer tests were performed on the volar aspect of the forearm by creating a negative pressure of $20 \mathrm{~mm} \mathrm{Hg}$ for $60 \mathrm{sec}$ beneath a circular glass cup with an internal diameter of $1.3 \mathrm{~cm}$. The number of petechiae produced in the circular area was counted. Normal adults show $0-1$ petechiae.

\section{Platelet Adhesiveness}

Native blood platelet adhesiveness was determined by a modification of the method of SALzMaN [19]. Venous blood, $1.5 \mathrm{ml}$, was drawn into a plastic syringe; $0.5 \mathrm{ml}$ was immediately placed in a tube containing ethylenediamine tetraacetate (EDTA) anticoagulant. The remaining $1.0 \mathrm{ml}$ of blood was passed through a column containing $1 \mathrm{~g}$ of glass beads [24] in exactly $25 \mathrm{sec}$ by means of a portable pump and collected in another tube containing EDTA anticoagulant. Platelet counts were performed on the 'before' and 'after' samples and the percentage of adhesive platelets was calculated.

\section{Thrombelastogram}

Thrombelastograms were done on recalcified citrated samples of whole blood by the standard technique. The thrombelastograph is an instrument for the measurement of the coagulability of whole blood or plasma. The $\mathrm{r}+\mathrm{k}$ measurement $(\mathrm{min}$ ) is the length of time required for initial clot formation. The ma measurement $(\mathrm{mm})$ reflects the tensile strength and durability of the clot.

\section{Platelet Aggregation}

Platelet aggregation with adenosine diphosphate (ADP), collagen, and thrombin was studied by turbimetric methods [16]. After performing a platelet count on 1 aliquot of PRP, the final platelet count was adjusted to $100,000 / \mathrm{mm}^{3}$ by diluting the PRP with the PFP of the patient and $0.35 \mathrm{ml}$ of veronal buffer, $\mathrm{pH}$ 7.34. The volume of the final test mixture of PRP, PFP, and veronal buffer was $0.7 \mathrm{ml}$.

Dilutions of ADP [25] were made with veronal buffer. Bovine thrombin [26] dilutions were made 
with $50 \%$ glycerol and water and were stored at $-30^{\circ}$. Rat tendon collagen was obtained commercially [27]. One gram of the dried material was suspended in $30 \mathrm{ml}$ Tyrode's solution and homogenized in a blender for $10 \mathrm{~min}$. The mixture was then centrifuged at $950 \times \mathrm{g}$ for $20 \mathrm{~min}$ at $0^{\circ}$. The supernatant was removed and diluted with Tyrode's solution. The storage stability of each of the three aggregating agents was evaluated by weekly checks of the reproducibility of platelet aggregation curves of the same normal adult controls.

A modified titrator [28] coupled to a recorder [29] was used for studying changes in turbidity. With the addition of ADP, collagen, or thrombin, the optical density (OD) of the PRP progressively decreased as the platelets aggregated. Platelet aggregation with each of the aggregating agents was characterized as time in seconds required for maximal platelet aggregation to occur ( $\mathrm{T} \triangle \mathrm{OD} \max$ ), and as percentage of total change in optical density. The percentage total platelet aggregation was directly related to the percentage of total optical density change. One hundred percent platelet aggregation was equated with a decrease in the optical density from $0.4 \mathrm{OD}$ to $0.0 \mathrm{OD}$ units.

Platelet aggregation with each of the three different aggregating agents was studied in each patient when sufficient PRP was available. Adenosine diphosphate concentrations given in the text refer to the final concentration of ADP in the experimental mixture. When thrombin was used as the aggregating agent, 0.15 of a unit was added to the PRP mixture by means of a micrometer. In the collagen-aggregating studies, 0.2 $\mathrm{ml}$ of a 1:6 Tyrode's suspension of collagen was the usual amount added.

Platelet aggregation with varying concentrations of ADP and collagen was studied on the cord blood of 10 normal infants and of 10 normal adults. The concentrations of ADP used were 13, 26, 52, and 520 $\mu \mathrm{moles} / \mathrm{ml}$. Collagen suspension $(0.2 \mathrm{ml})$ was added undiluted, and diluted 1:4, or 1:6 with Tyrode's solution.

Mixing experiments, in which platelets from infants were suspended in adult PFP or adult platelets were suspended in infant PFP, were done as follows. In experiment 1 , adult PRP was prepared as previously described. The PRP was then centrifuged at $600 \times g$ for 20 min and the PFP was removed leaving only the platelet button. The adult platelets were then gently resuspended in an equal volume of infant PFP and a platelet count was performed. Appropriate dilution of the PRP with infant PFP and veronal buffer was done to obtain a final platelet count of $100,000 / \mathrm{mm}^{3}$. Platelet aggregation with ADP was done on one sample immediately and on another sample after incubation for $60 \mathrm{~min}$ at $37^{\circ}$. Experiment 2 was conducted exactly like experiment 1 except that the infant platelets were resuspended in normal adult PFP.

Experiment 3 was designed to detect the presence of an ADP inhibitor in plasma from infants. One 0.35$\mathrm{ml}$ aliquot of adult PFP and three $0.35-\mathrm{ml}$ aliquots of infant PFP were prepared; ADP was added to each of the four tubes resulting in a final concentration of 26 $\mu$ moles $\mathrm{ADP} / \mathrm{ml}$ in the final plasma mixture. Each of the tubes of infant PFP with added ADP was allowed to incubate at $37^{\circ}$ in a water bath for $0.25,2$, and $6 \mathrm{~min}$, respectively. At the end of the designated incubation period, $0.35 \mathrm{ml}$ of adult PRP was added to each tube. Platelet aggregation was recorded as described.

Additional experiments were done on PRP from cord blood of three normal term infants to determine whether the previously observed impairment of platelet aggregation was related to a biased sampling of a specific platelet population since platelet function can be different depending upon the size and age of the platelet [13]. Infant PRP and PFP was prepared in the usual manner. A $0.5-\mathrm{ml}$ aliquot of PRP was removed and replaced with $0.5 \mathrm{ml}$ of PFP from the same infant. The original tube containing citrated whole blood with the supernatant PRP was then inverted 10 times and recentrifuged at $130 \times g$ for $20 \mathrm{~min}$ at $4^{\circ}$. A second aliquot of PRP was removed and the entire procedure was repeated two additional times until a total of four PRP aliquots had been harvested from the citrated whole blood of the same infant. Adenosine diphosphate platelet aggregation was then performed on each aliquot.

\section{Fibrin-split Products}

Fibrin-split products (FSP) were measured in infant and adult serum samples by the qualitative capillary tube precipitation technique of GLAMAN and MERRILL [4] in which rabbit anti-human fibrinogen antiserum was used instead of antisera to immunoglobulins. Thrombinized serum of the patients was collected in the presence of a proteinase inactivator, Trasylol [30].

Studies to determine the in vitro effect of exogenous fibrin-split products on ADP and thrombin-induced platelet aggregation were performed on adult PRP. Fibrin-split products, composed predominately of the smaller fragments $\mathbf{D}$ and $\mathbf{E}$, were prepared by incubation of human fibrinogen [31] with fibrinolysin [32] for $4 \mathrm{~h}$ at $37^{\circ}$; the reaction was stopped by adding soybean trypsin inhibitor. Varying dilutions were made with veronal buffer so that the final concentrations of FSP in the PRP mixtures were as follows: $12.5,5,2.5$, 0.25 , and $0.025 \mathrm{mg} / \mathrm{ml}$. The studies of ADP and thrombin platelet aggregation were performed on adult PRP containing each of the various concentrations of FSP. 


\section{Platelet Factor 3}

Platelet factor 3 assays were performed using the method of Horowitz and Papayoanou [11] on the cord blood of 10 normal infants and on blood from 10 normal adults. A decrease in the Stypven time of at least $10 \mathrm{sec}$ following incubation with ADP was considered a normal test for release of platelet factor 3 .

\section{Platelet Fibrinogen}

Platelet fibrinogen was determined on the cord blood of a full-term infant and on an adult according to the method outlined by CAstald and CAEN [5]. All the wash fluids and extracts were tested for the presence of fibrinogen with latex-antifibrinogen [33]. One drop each of the latex-antifibrinogen and platelet wash or extract were mixed together on a glass slide and the time of agglutination recorded. The agglutination time of the mixture was approximately proportional to the concentration of fibrinogen present.

The results of the various platelet function studies performed on both infants and adults are expressed as mean values \pm standard error of the mean (SEM). Probability correlation was determined by Student's $t$ test.

\section{Results}

Bleeding Times, Clot Retraction, and Petechiometer Tests

There was no significant difference in the bleeding times between the normal term infants and the normal adults (table I). In whole blood of normal term infants, clot retraction was grossly defective when compared with normal adult values. Normal term infant PRP clot retraction studied in five infants was also greatly impaired. The petechiometer tests were negative in both the adult and normal term infant groups. Bleeding times, clot retraction, and petechiometer tests were not performed on a sufficient number of the premature infants for statistical comparison.

\section{Platelet Adhesiveness and Thrombelastograms}

There was no significant difference in platelet adhesiveness between the adult group and any of the infant groups (table $\mathrm{I}$ ). The $\mathrm{r}+\mathrm{k}$ measurement of the thrombelastogram was significantly short $(P<0.01)$ in the normal term infants when compared with adult values. The ma measurement of the thrombelastogram was significantly greater $(P<0.002)$ in the term infants compared with the adult controls.

Platelet Aggregation with ADP, Collagen, and Thrombin

Figure 1 shows representative tracings of the platelets of a normal infant and of a normal adult aggregating with ADP. The time required for maximal platelet aggregation to occur (T $\Delta \mathrm{OD}$ max) for the infant was
$160 \mathrm{sec}$ compared with $120 \mathrm{sec}$ for the adult. Total percentage platelet aggregation was $50 \%$ for the infant and $80 \%$ for the adult.

Table II summarizes the results of the platelet aggregation studies in the adult and infan ${ }^{+}$groups. Aggregation studies with ADP revealed a significant prolongation in the time required for maximal platelet aggregation in the various infant groups. Also, the percentage total platelet aggregation in the infant groups was significantly less than that of the normal adult group. There was significant difference among the four groups of infants studied neither with regard to total platelet aggregation with ADP nor to time required for maximal optical density change. Comparison of normal premature infants and premature infants with RDS or apneic spells with normal adults showed a significant difference $(P<0.001)$ for total platelet aggregation with ADP, but none $(P<0.30)$ with regard to time required for maximal optical density change. Similar results were obtained in a few infants when heparin ( 1 unit/1 ml blood) was used as an anticoagulant.

Platelet aggregation with collagen showed that the time required for maximal platelet aggregation was significantly longer $(P<0.001)$ in normal term infants than in normal adults. The T $\triangle \mathrm{OD}$ max was $443 \mathrm{sec}$ for the infants and $230 \mathrm{sec}$ for the adults. The total platelet aggregation to collagen was significantly less $(P<0.001)$ in the infants than in the adults $(73$ versus $91 \%)$.

As with the other two aggregating agents, normal term infants showed impaired platelet aggregation with thrombin. The time required for maximal platelet aggregation was significantly longer, and the total platelet aggregation was significantly less in this group when compared with the adult group.

Figures 2, 3, and 4 are composite plots of platelet aggregation to ADP, collagen, or thrombin of normal adults and normal term infants. Regardless of the aggregating agent, it can be seen that normal adults have a higher total percentage platelet aggregation than term infants, and the time required for maximal platelet aggregation is considerably shorter than that of the infants. Four infants with $0 \%$ total platelet aggregation and maximal aggregation times of greater than $280 \mathrm{sec}$ are illustrated in figure 2. These four infants are not the same four infants shown in figure 4 who had prolonged maximal aggregation times with thrombin.

\section{Platelet Aggregation with Varying Concentrations of ADP and Collagen}

The results of various concentrations of ADP (13-520 $\mu \mathrm{moles} / \mathrm{ml}$ PRP) on platelet aggregation in samples from 10 normal adults and 10 normal term infants 
Altered platelet function in newborns
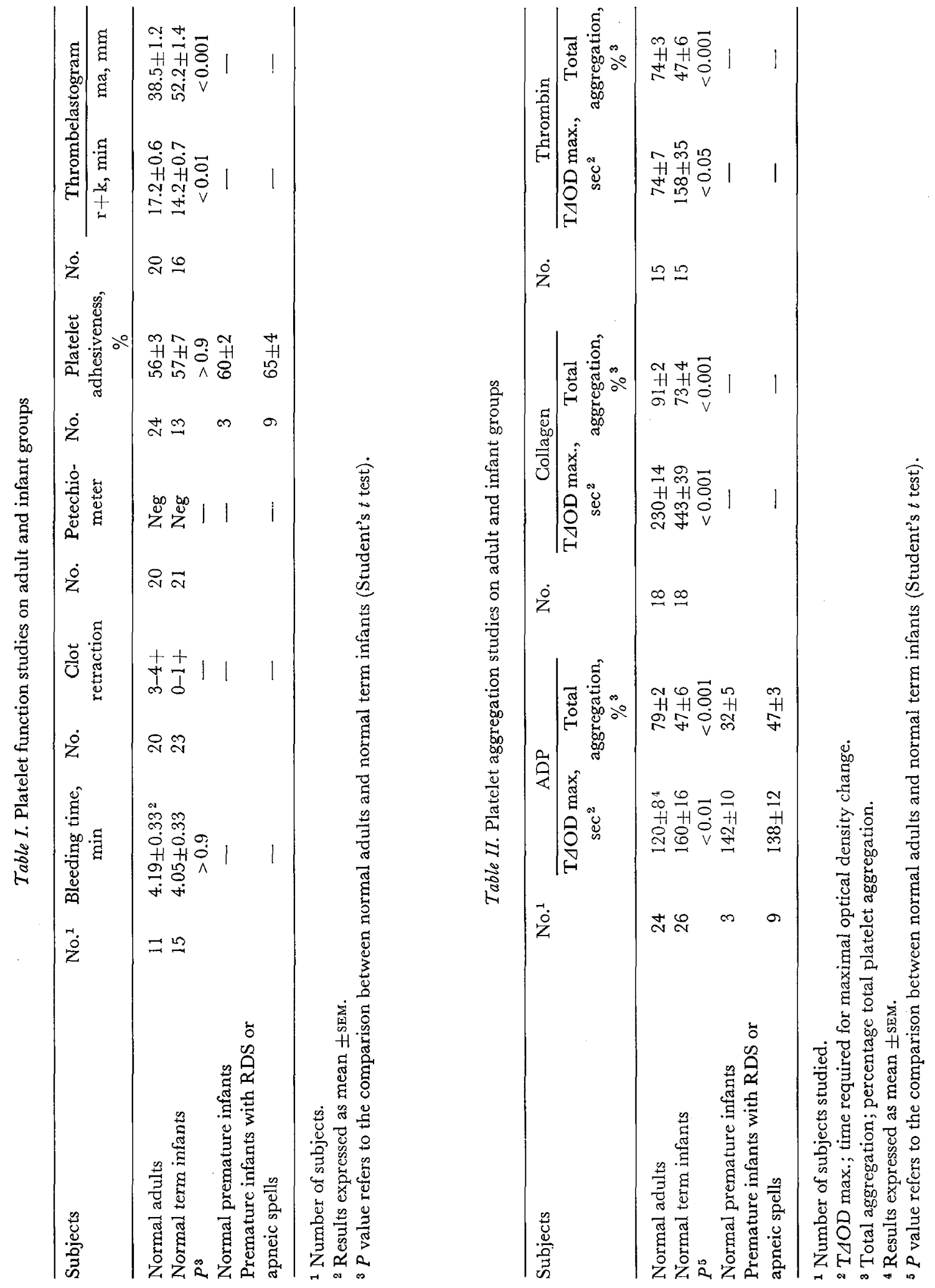
showed that platelet aggregation with ADP from infant samples remained defective compared with aggregation from normal adult samples, even when concentrations of ADP 20 times greater than usual were employed.

Similarly, the effect of three different concentrations of collagen $(1: 1,1: 4,1: 6$ dilutions $)$ on platelet aggregation in samples from 10 normal term infants and 10 normal adults showed that infant platelet aggregation remained abnormal when compared with that of normal adults regardless of collagen concentration.

\section{Platelet Aggregation Mixing Experiments}

Adenosine diphosphate platelet aggregation following resuspension of infant platelets in adult PFP, or adult platelets in normal infant PFP, revealed that adult platelets aggregated normally whether resus-

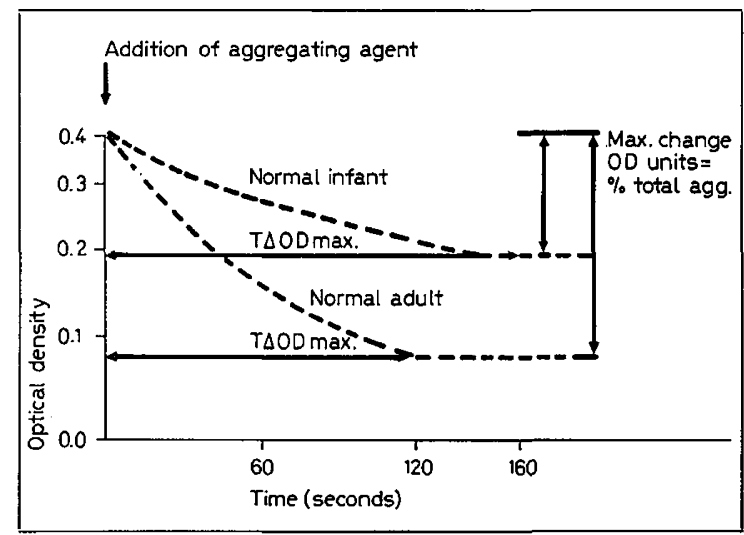

Fig.1. Representative aggregrometer tracings of platelet aggregation with ADP of normal infant and normal adult.

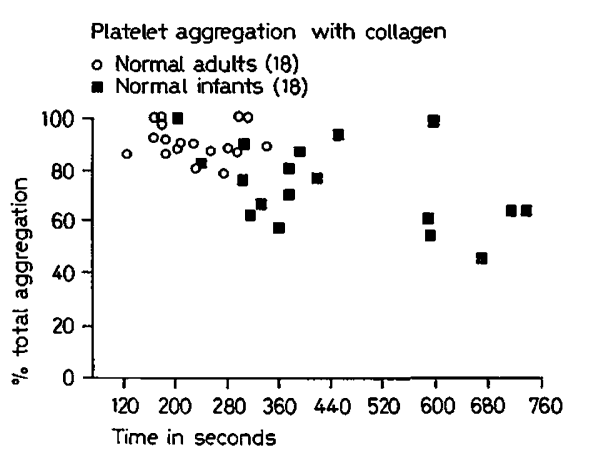

Fig.3. Composite plot of platelet aggregation with collagen of normal adults and normal infants. pended in normal adult PFP or in normal term infant PFP; in contrast, infant platelet aggregation to ADP remained defective whether the platelets were resuspended in normal infant PFP or normal adult PFP. Incubation of either the infant or adult platelets in their own or opposite PFP did not appear to alter response to ADP.

Adenosine diphosphate incubated for various periods of time in infant plasma did not lessen significantly the subsequent aggregation of added adult platelets; therefore, the presence of an ADP inhibitor in infant plasma seems unlikely.

Platelet aggregations with ADP in four separate serial aliquots of PRP harvested after repeated mixing and recentrifugation of citrated whole blood from normal term infants failed to show any improvement in the infant platelet aggregating defect.

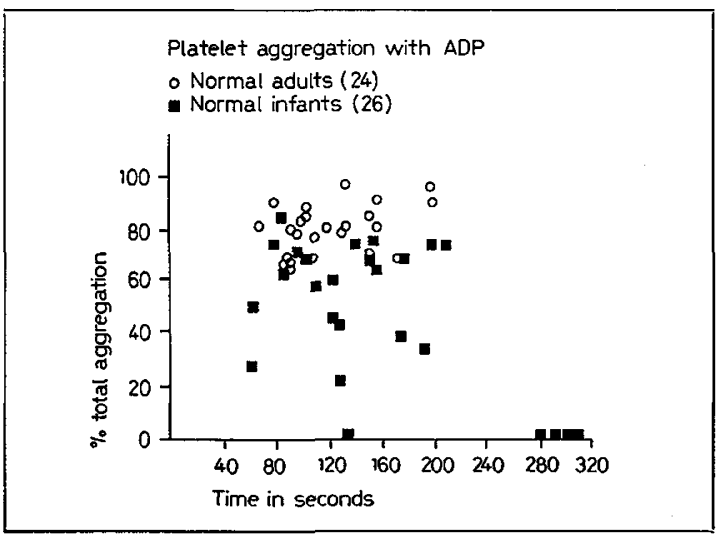

Fig.2. Composite plot of platelet aggregation with ADP of normal adults and normal infants.

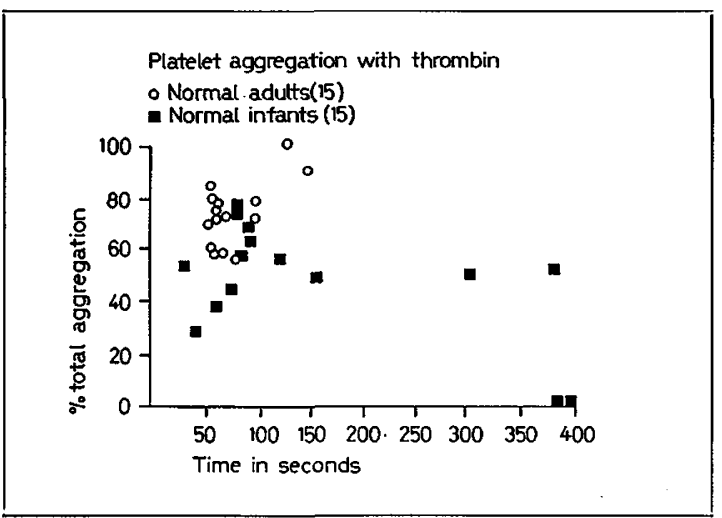

Fig.4. Composite plot of platelet aggregation with thrombin of normal adults and normal infants. 


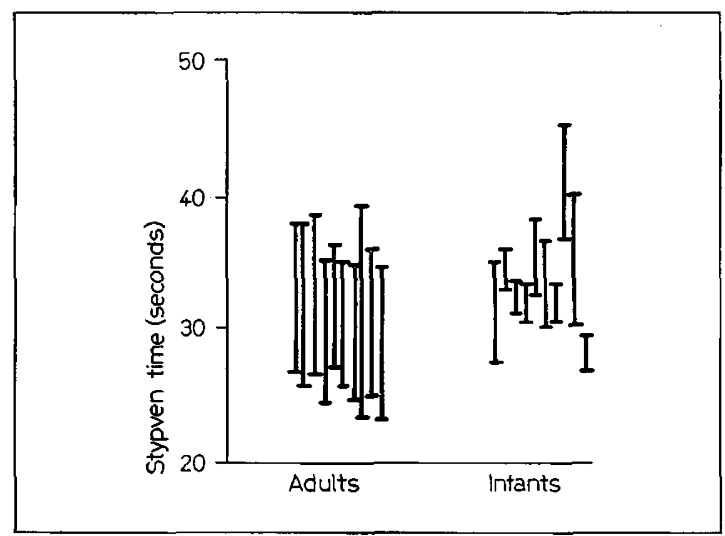

Fig.5. Platelet factor 3: the length of each bar represents the difference between the Stypven time before and after incubation with ADP. The longer the bar, the greater the platelet factor 3 activity.

\section{Fibrin-split Products}

Fibrin-split products were determined in 13 normal term infants who exhibited abnormal platelet aggregation. The serum of five of these infants showed trace concentrations of FSP.

The addition of FSP to normal adult PRP in vitro did not impair platelet aggregation to either ADP or collagen.

\section{Platelet Factor 3}

Figure 5 illustrates the difference in platelet factor 3 activation between 10 normal adults and 10 normal term infants. These findings suggest that platelets from normal infants have decreased platelet factor 3 activity.

\section{Platelet Fibrinogen}

The results of assays for platelet fibrinogen from a normal term infant and a normal adult indicated no significant difference in the amount of platelet fibrinogen present in the two subjects.

\section{Discussion}

Platelet function in the newborn infant has been found to be altered or impaired when compared with platelet function in normal adults. The most striking defects noted in normal term infants were defective whole blood and PRP clot retraction, impaired platelet aggregation to ADP, collagen, and thrombin, and decreased platelet factor 3 activity. Other variables of platelet function in the term infants such as bleeding time, petechiometer test, and adhesiveness to glass beads did not differ significantly from those of normal adult controls. Studies of platelet function in premature newborn infants and sick newborns are insufficient in number at this time to determine if these high risk infants have altered platelet function of equal or greater magnitude than normal term infants.

Hrodek, in a recent monograph [12], has noted the sparsity of studies of platelet function in the newborn. His studies showed 1) an impaired aggregation of platelets when exposed to glass, 2) defective adhesiveness of platelets to glass fibers, 3) decreased maximal amplitude of the thrombelastogram, 4) low platelet factor 3, and 5) normal clot retraction and antiheparin platelet activity. Using different methodology, our studies agree with those of HRODEk with the exception that we found 1) a normal whole blood platelet adhesiveness, 2) an increased ma on the TEG, and 3) impaired clot retraction. These discrepancies may be explainable on the basis of methodology. The normal adhesiveness of platelets to glass that we obtained could be related to the high hematocrit seen in the newborn [9]. The hypercoagulability of newborn blood (short $\mathrm{r}+\mathrm{k}$, increased ma on the TEG), which is only inferentially related to platelet function, has been previously reported by von KaUlla and ButTERField [22], and Markarian et al. [15]. Hilgartner [10] also has found impaired clot retraction in the newborn as well as impaired platelet factor 3 release and ADP platelet aggregation.

Bleeding times have been reported by most authors to be within normal limits $[1,12,20]$. The explanation and significance of these alterations in platelet function in the newborn infant remains to be determined.

Impaired platelet aggregation to ADP, collagen, and thrombin has not been previously reported in newborn infants. This defect could not be corrected or overcome by utilizing increasing concentrations of ADP or collagen. The role of the citrate anticoagulant was controlled by using a uniform amount of citrate as determined by the hematocrit. Heparinized PRP also showed impaired aggregation to ADP. Selections of different populations of platelets by repeated centrifugations of the blood did not yield significant differences in the results. Mixing experiments, in which infant platelets were resuspended in adult PFP, neither improved the platelet aggregating response of the infant to ADP, nor did adult platelets resuspended in infant PFP lose their ability to aggregate normally with ADP. A plasma inhibitor of ADP could not be demonstrated. It appears unlikely from these experiments that impaired ADP platelet aggregation of newborns was due to the presence of plasma ADP inhibitors, a refractory state due to increased circulating levels of ADP, anticoagulants, or a selected population of platelets.

It has been suggested that platelet function can be impaired in the presence of increased circulating levels of fibrin-split products [14]. Since newborns are known 
to have increased fibrinolytic activity, it was considered important to determine if they had detectable FSP in their serum which could be the basis for their impaired platelet aggregation. In a previous study, FSP were found in the serum of $18 \%$ of normal newborns [8]. Only one-third of these infants had impaired platelet aggregation. Our studies, using the in vitro addition of FSP to PRP, did not indicate that fibrinsplit products interfered with platelet aggregation. These studies are in agreement with those of CRONBERG [6]; therefore, it seems unlikely that circulating FSP were responsible for defective platelet function, although the influence of platelet-bound FSP cannot be eliminated.

The presence of white blood cells in PRP has been reported to have an inhibitory effect on ADP-induced platelet aggregation [7]. The lowest effective concentration of cells was found to be $2,000 / \mathrm{mm}^{3}$ or greater. White blood cell (WBC) counts on PRP from 10 different subjects in our study failed to show a WBC count greater than $250 / \mathrm{mm}^{3}$.

A deficiency of platelet fibrinogen has been implicated as the cause of impaired platelet aggregation in thrombasthenic patients [17]. Evidence of a deficiency of extractable platelet fibrinogen could not be demonstrated in the platelets of the newborn infant.

Evidence at the present time suggests that impaired platelet function seen in normal newborns presumably reflects a transitory defect in the platelets rather than an absent or inhibitory plasma component. These 'physiologic' alterations closely resemble defective platelet functions seen in patients with Glanzmann's thrombasthenia [3]. Platelet survival in vivo, and metabolic and ultrastructural studies of the platelet of the newborn should clarify the cause and significance of these physiologic findings.

It should be emphasized that although altered platelet function was documented in the study infants, none of them showed a bleeding tendency. The relation of these findings to increased susceptibility to hemorrhage or to protection against intravascular thrombosis remains to be determined.

\section{Summary}

The results of studies of platelet function performed on 64 newborn infants have been presented and values compared with those found in normal adult controls. The infants showed altered or impaired platelet function with regard to clot retraction, platelet aggregation to ADP, collagen, and thrombin, and decreased platelet factor 3 activity. Thrombelastograms were compatible with hypercoagulability of the blood. Other accepted variables of platelet function such as bleeding time, petechiometer test, and platelet adhesiveness to glass beads were normal.

None of the infants studied had clinical evidence of a bleeding tendency. The altered platelet function was probably due to a transitory defect in the platelct rather than an absent or inhibitory plasma factor.

\section{References and Notes}

1. Aballi, A.H. and Delamerens, S.: Coagulation changes in the neonatal period and in early infancy. Pediat. Clin. N. Amer. 9: 785 (1962).

2. Brecher, S. and CRonkite, E.P.: Morphology and enumeration of human blood platelets. J. appl. Physiol. 3: 365 (1960).

3. Caen, J.P.; Castaldi, P.A.; Leclerc, J. G.; Inceman, S.; Larrieu, M.J.; Probst, M. and BerNARD, J.: Congenital bleeding disorders with long bleeding time and normal platelet count. I. Glanzmann's thrombasthenia (report of fifteen patients). Amer.J.Med. 41: 4 (1966).

4. Calaman, H.N. and Merrill, D.: Quantitative measurements of human gamma-2, beta-2A and beta-2N serum immunoglobulins. J.Lab.clin. Med. 64: 685 (1964).

5. Castaldi, P.A. and Caen, J.: Platelet fibrinogen. J.clin. Path. 18: 579 (1965).

6. Cronberg, S.: Effect of fibrinolysis on adhesion and aggregation of human platelets. Thromb. Diath. haemorrh. 19: 474 (1968).

7. Harrison, M.J.G.; Emmons, P.R. and Mitchell, J.R.A.: The effect of white cells on platelet aggregation. Thromb. Diath. haemorrh. 16: 105 (1966).

8. Hathaway, W.E.; Mull, M.M. and Pechet, G.S.: Disseminated intravascular coagulation in the newborn. Pediatrics 43: 233 (1969).

9. Heliem, A.J.: The adhesiveness of human blood platelets in ritro. Scand. J. clin. Lab. Invest. Suppl. 51 (1960).

10. Hilgartner, M.W.: Transient functional thrombasthenia in the newborn (Abstract). Society for Pediatric Research, 38th Annual Meeting, 1968, p. 120.

11. Horowitz, H. E. and Papayoanou, M. F. : Activation of platelet factor 3 by adenosine $5^{\prime}$ diphosphate. Thromb. Diath. haemorrh. 19: 18 (1968).

12. Hrodek, O.: Blood platelets in the newborn: Their function in haemostasis and hemocoagulation. Acta Univ. Carolinae Monogr. 22 (1966).

13. Karpatkin, S.: Heterogeneity of human platelets. 1. Metabolic and kinetic evidence suggestive of young and old platelets. J.clin. Invest. 48: 1073 (1969).

14. KoWAlski, E. ; Kopec, M. and Wegrzynowicz, Z. : 
Influence of fibrinogen degradation products on platelet aggregation, adhesiveness, and viscous metamorphosis. Thromb. Diath. haemorrh. 10: 406 (1964).

15. Markarian, M.; Jackson, J.; Bannon, A. and Rosenblut, E.: Evidence of hypercoagulability in premature infants with the respiratory distress syndrome (RDS) (Abstract). Society for Pediatric Research, 36th Annual Meeting, 1966, p. 123.

16. Mustard, J.R.; Hegardt, B.; Rowsell, H.C. and MAcMrllan, R.L.: The effect of adenosine nucleotides on platelet aggregation and clotting time. J. Lab. clin. Med. 64: 548 (1964).

17. Nachman, R.L.: Thrombasthenia: Immunologic evidence of a platelet protein abnormality. J.Lab. clin. Med. 67: 411 (1966).

18. Oski, F.A. and Naiman, M.L.: in: A.J.Schaffer: Hematologic problems in the newborn (Saunders, Philadelphia/London 1966).

19. Salzman, E.W.: Measurement of platelet adhesiveness. J.Lab. clin. Med. 62: 724 (1963).

20. SANFORD, H.N. and SHMigelsky, Z.: Quantitative and qualitative platelet values of normal newborn infants. Amer. J. Dis. Ghild. 63: 729 (1942).

21. Sutherland, J.M.; Glueak, H.E. and Gleser, G. : Hemorrhagic disease of the newborn. Amer.J. Dis. Child. 133: 524 (1967).

22. von Kaulla, K.N.; von Kaulla, E. and ButterFIELD, J.: Fibrinolytic activity, thrombin inhibitor, and kinetics of clot formation in premature infants with the respiratory distress syndrome. Acta paediat. scand. 54: 587 (1965).

23. Informed consent was obtained for all subjects in this study.

24. Superbrite glass beads, type 070, 3M Company, Minneapolis, Minn.

25. Calbiochem, Inc., Los Angeles, CA.

26. Upjohn and Company, Kalamazoo, MI.

27. Sigma Chemical Company, St. Louis, MO.

28. Evans Electroselenium Ltd., Halstead, Essex, England.

29. Model SR, Sargent-Welch Scientific Company, Skokie, IL.

30. Traysylol, FBA Pharmaceuticals, New York, NY.

31. Obtained from Cutter Laboratories, Berkeley, GA.

32. Obtained from the Michigan Department of Public Health, Lansing, MI.

33. Fi-Test, Hyland Laboratories, Los Angeles, CA.

34. We express our appreciation for technical assistance to Mrs. Judy Alsever.

35. Supported by Easter Seal Foundation Grant no. R-6637 and by Public Health Service Research Grant no. HD-01965-03.

36. Requests for reprints should be addressed to: Dr. MARILyn M.Mull, Department of Pediatrics, University of Colorado Medical Center, Denver, CO, 80220 (USA).

37. Accepted for publication November 6, 1969. 